\title{
How does contrasting dependence of impurity-atom diffusivity on the density of host disordered medium arise?
}

\author{
Manju Sharma and S Yashonath* \\ Solid Sate and Structural Chemistry Unit, Indian Institute of Science, Bangalore-560 012, India \\ E-mail : yashonat@sscu.iisc.ernet.in
}

Received 30 September 2008, accepted 6 December 2008

\begin{abstract}
We report results of molecular dynamics investigations into neutral impurity diffusing within an amorphous solid as a function of the size of the diffusant and density of the host amorphous matrix. We find that self diffusivity exhibits an anomalous maximum as a function of the size of the impurity species. An analysis of properties of the impurity atom with maximum diffusivity shows that it is associated with lower mean square force, reduced backscattering of velocity autocorrelation function, near-exponential decay of the intermediate scattering function (as compared to stretched-exponential decay for other sizes of the impurity species) and lower activation energy. These results demonstrate the existence of size-dependent diffusivity maximum in disordered solids. Further, we show that the diffusivity maximum is observed at lower impurity diameters with increase in density. This is explained in terms of the Levitation parameter and the void structure of the amorphous solid. We demonstrate that these results imply contrasting dependence of self diffusivity $(D)$ on the density of the amorphous matrix, $\rho$. $D$ increases with $\rho$ for small sizes of the impurity but shows an increase followed by a decrease for intermediate sizes of the impurity atom. For large sizes of the impurity atom, $D$ decreases with increase in $\rho$. These contrasting dependence arises naturally from the existence of Levitation Effect.
\end{abstract}

Keywords : Amorphous solid, anomalous diffusion, density impurity

PACS Nos. : 61.43.-j, 66.30.hh, 66.30.Ny

\section{Introduction}

Transport properties play an important role in a wide class of materials as well as in living systems. Self diffusivity predominantly determines the transport property; although distinct diffusivity makes contributions to transport diffusivity, it is usually not very large $[1,2]$. It is therefore necessary to understand how different factors modify self diffusivity. There have been innumerable investigations on the influence of different factors on self diffusivity [3-7].

A number of glasses exhibit changes in ionic conductivity with composition. 
For example, Pradel et al [7] have investigated conductivity as a function of composition in $x \mathrm{Li}_{2} \mathrm{O}-(1-x) \mathrm{P}_{2} \mathrm{O}_{5}$ and $x \mathrm{Li}_{2} \mathrm{~S}-(1-x) \mathrm{GeS}_{2}$ glasses. They find that conductivity exhibits a maximum as a function of composition. Hosono et al [8] have studied conductivity in $\mathrm{Agl}: \mathrm{Ag}_{2} \mathrm{WO}_{4}$ glasses and find that the conductivity varies as a function of composition. In these glasses, the density of the glass is a function of the composition.

Minami [9] has studied $\mathrm{Li}_{4} \mathrm{SiO}_{4}-\mathrm{Li}_{3} \mathrm{BO}_{3}$ glasses; the ionic conductivity of $\mathrm{Li}^{+}$varies as a function of the composition of $\mathrm{Li}_{3} \mathrm{BO}_{3}$ while at the same time no increase in the number of conducting $\mathrm{Li}^{+}$ions is seen, clearly suggesting that this increased conductivity arises from changes in the amorphous matrix in which $\mathrm{Li}^{+}$ions diffuse.

There have been a few studies on diffusivity in melts of silica and related substances. Woodcock, Angell and Cheeseman [10] found that glasses of the type MX and $\mathrm{MX}_{2}$ (e.g., $\mathrm{KCl}, \mathrm{BeF}_{2}, \mathrm{ZnCl}_{2}$ and $\mathrm{SiO}_{2}$ ) exhibit a maximum in diffusivity of the constituent ions at intermediate densities. Angell, Cheeseman and Tamaddon [11] discovered that in simulated silicate melt, $\mathrm{NaAlSi}_{2} \mathrm{O}_{6}$, ions such as $\mathrm{Si}^{4+}, \mathrm{Al}^{3+}$ as well as $\mathrm{O}^{2-}$ exhibit a maximum between 200 and 300 kilobars.

In another series of simulation studies we have carried out, it has been found that self diffusivity does not exhibit a simple monotonic dependence on the size of the diffusant in dense liquids [12]. Instead, a maximum in self diffusivity has been found as a function of the solute size. Recently, we have shown that this leads to breakdown in the Stokes-Einstein expression [13] : self diffusivity does not exhibit a simple inverse relationship with the solute size over the whole range of solute size. Instead, over a certain range of the solute size, the self diffusivity of the solute is higher than what is given by the Stokes-Einstein expression. The absence of attractive $Q$ interaction leads to no such enhanced self diffusivity has been discussed in a recent article [2] thereby demonstrating that this enhanced diffusivity has its origin in the dispersion (or the attractive part of the van der Waals interaction) between the solute and solvent. Further, we have also shown that the important quantity of interest is the solute-solvent radius ratio. Thus the maximum in self diffusivity occurs for a solute size that is determined by the solvent. This is, however, only an approximate statement. A more precise quantity is the ratio of solute radius at which the optimum interaction occurs with the solvent to the neck radius of pore network in the liquid through which the solute diffuses.

Although the density of these glasses changes with composition, and the change in density is one possible cause of the changes in ionic conductivity, there have been no detailed investigation into dependence of self diffusivity in glasses. Here we report molecular dynamics simulations of impurity atom in an amorphous matrix. We examine whether similar diffusivity maximum exists in dense amorphous solids interacting via van der Waals interactions. Our results suggest that a diffusivity maximum exists even in amorphous solids. There is a particular size of the impurity species for which the 
self diffusivity is maximum. Further, we carry out simulations at several densities and show how the size dependence of self diffusivity is altered. We derive the relation between the self diffusivity and density of the amorphous matrix : three contrasting relationships are seen depending on the size of the impurity atom. We then explain this dependence based on the previous results and understanding of the Levitation Effect.

\section{Model and intermolecular potential}

Calculations on the host-impurity system have been performed at three different densities. Configuration of amorphous host atoms was prepared from simulations in the microcanonical ensemble with cell length chosen to correspond to the desired density in the following way. Host particles are arranged on a $f c c$ lattice to begin with and heated to high temperature. The resulting melt is then quenched to low temperature to obtain an amorphous solid. Host-host interactions are modelled in terms of LennardJones potential with parameters specified in Table 1 ,

$$
\phi=4 \in\left[\left(\frac{\sigma}{r}\right)^{12}-\left(\frac{\sigma}{r}\right)^{6}\right] .
$$

Subsequent simulations were performed with this host configuration. Host configuration is assumed frozen and only the impurity atoms are included in the molecular dynamics integration for runs to estimate the self diffusivity and other properties whose results are discussed in next sections.

\begin{tabular}{|c|c|c|}
\hline Type of interaction & $\sigma, \AA$ & $\in, \mathrm{kJ} / \mathrm{mol}$ \\
\hline$h h$ & 4.1 & 4.0 \\
\hline$i h$ & $1.0-2.1$ & 3.0 \\
\hline$i i$ & $0.3-\sigma_{m}$ & 0.99 \\
\hline
\end{tabular}

Voronoi tessellation of the final equilibrated configuration of this amorphous phase yielded the void and neck distributions as well as the position of the void centers. Impurity atoms are assigned to the randomly chosen void centers obtained from the Voronoi tessellation.

Host-impurity as well as impurity-impurity interactions are modelled in terms of Lennard-Jones potential. The impurity-impurity Lennard-Jones diameter, $\sigma_{i i}$ is varied over a range (see Table 1). The host-impurity diameter, $\sigma_{i h}=\sigma_{i i}+0.7 \AA[14,15]$.

The impurity-host, $U_{i h}$ and impurity-impurity, $U_{i i}$ interaction energy constitutes the total interaction energy of the system, $U_{\text {tot }}$, 


$$
U_{\text {tot }}=U_{i h}+U_{i i}
$$

\section{Computational details}

We have chosen to work at three reduced densities : $\rho^{*}=N_{h} /\left(V / \sigma_{h h}^{3}\right)$ of $0.933,1.05$ and 1.187. Here $V=L^{3}$ where $L=33.3 \AA$ for $\rho^{*}=0.933$. There are in all $5324\left(N_{h}\right)$ host atoms and 600 impurities $\left(N_{i}\right)$ at each density. We note that there are approximately $3 N_{h}$ voids in an amorphous solid and therefore the number of impurity atoms are lower than the number of voids. We note that the results presented here remain valid for a rather wide range of impurity atom concentration (1-30\%). The mass of host and impurity atoms has been chosen to be 85 and 40 amu respectively. All simulations have been carried out with Verlet leapfrog algorithm using DLPOLY [16] using cubic periodic boundary conditions. An initial crystalline fcc host configuration is heated to $10000 \mathrm{~K}$ and the system is equilibrated for $10 \mathrm{ps}$ (timestep, $1 \mathrm{fs}$ ). This is followed by a production run of $10 \mathrm{ps}$ with no velocity scaling. The system is then quenched to $60 \mathrm{~K}$ and equilibrated for $300 \mathrm{ps}$ (timestep, $5 \mathrm{fs}$ ). This gives us the final configuration of the host amorphous matrix which is subsequently used for all simulations at that density. Runs have been made at $60 \mathrm{~K}$ except for calculation of activation energy when runs at a few other temperatures were made $(80,100$ and 120 $\mathrm{K})$. The host lattice has been treated as rigid in the simulations.

A timestep of 2 fs for impurity size $0.3 \AA$ and 5 fs for all other impurity sizes yielded a good energy conservation. The system is equilibrated for 500 ps and production run of 2 ns was made for all the sizes. Properties are then computed from positions, velocities and forces stored every $250 \mathrm{fs}$. The Voronoi tessellation analysis of the host configuration is carried out to obtain the void and neck distributions.

\section{Results and discussion}

The host-host radial distribution function for the three densities is reported in Figure 1. The split second peak is present for all three densities. This suggests that the packing

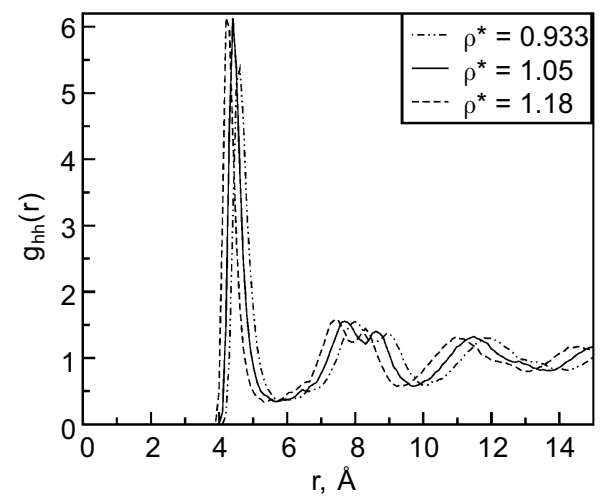

Figure 1. Host-host radial distribution function of the host configuration at the three densities. Note the split second peak which suggests that the configurations correspond to amorphous phase. 
is amorphous. All the three peaks shift to smaller distances with increase in density as expected.

In the present case, the impurity atoms are significantly smaller than the host atoms. Under these circumstances, impurity atom diffusion occurs through jumps from one interstitial to another. It is therefore of considerable utility to know the structure of void space in this solid. While structure of glassy solid as given by the radial distribution functions are also of importance, structure of the void space will provide greater insight as suggested by previous studies $[2,12]$. The neck is the interconnecting region between any two voids in the medium. Impurity diffuses from one void to another, through such a neck which is the bottleneck for diffusion. The void and neck distribution in the host medium can be obtained from Voronoi tessellation analysis. This is shown in Figure 2. The distribution shifts gradually towards smaller radii with increase in density. The width of void distribution decreases with increase in density :
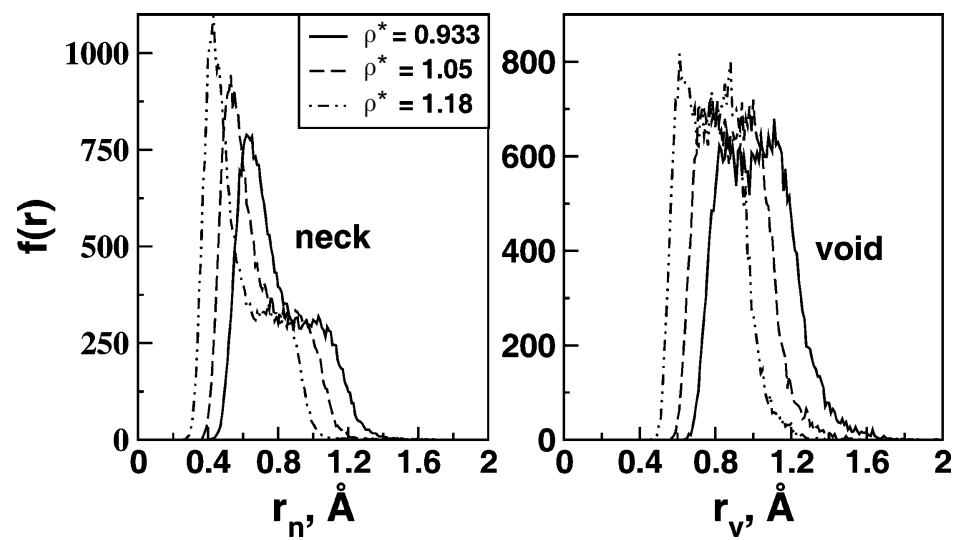

Figure 2. Neck and void distribution derived from a Voronoi polyhedral analysis of the host atom coordinates for reduced densities, $\rho^{*} 0.933,1.05$ and 1.187 .

widths are (0.65-1.65), (0.55-1.47), (0.4-1.28) $\AA$ for $\rho^{*}=0.933,1.05$ and 1.187 respectively. The width of neck radii extends over the range (0.44-1.4), (0.35-1.2), $(0.28-1.08) \AA$ at $\rho^{*}=0.933,1.05$ and 1.187 respectively. Similarly, the first peak maximum shifts to smaller distances and the width decreases with increase in the density for neck distribution. We shall see that this has significant influence on the variation of self diffusivity with the size of the impurity atom.

Average void, $\bar{r}_{v}$ and neck, $\bar{r}_{n}$ radii have been obtained from

$$
\begin{aligned}
& \bar{r}_{n}=\frac{\sum f_{n}(r) r}{\sum f_{n}(r)}, \\
& \bar{r}_{v}=\frac{\sum f_{v}(r) r}{\sum f_{v}(r)} .
\end{aligned}
$$


The average void radius, $\bar{r}_{v}$ and neck radius, $\bar{r}_{n}$ computed from distributions shown in Figure 2 for densities $0.933,1.05$ and 1.187 are $1.02,0.90,0.78 \AA$ and $0.79,0.69$, $0.57 \AA$ A respectively.

There is a significant change in void and neck distribution with increase in the density. We have investigated the effect of these changes on the diffusivity of impurities. The mean square displacement of the impurities at different densities was calculated. Figure 3 reports the mean square displacement plots for the three densities. The statistics is good as can be seen from the straight lines for mean square displacement. Initially, slope decreases with increase in the size of impurity. But for still larger sizes of the impurity species, an increase in the slope is seen.
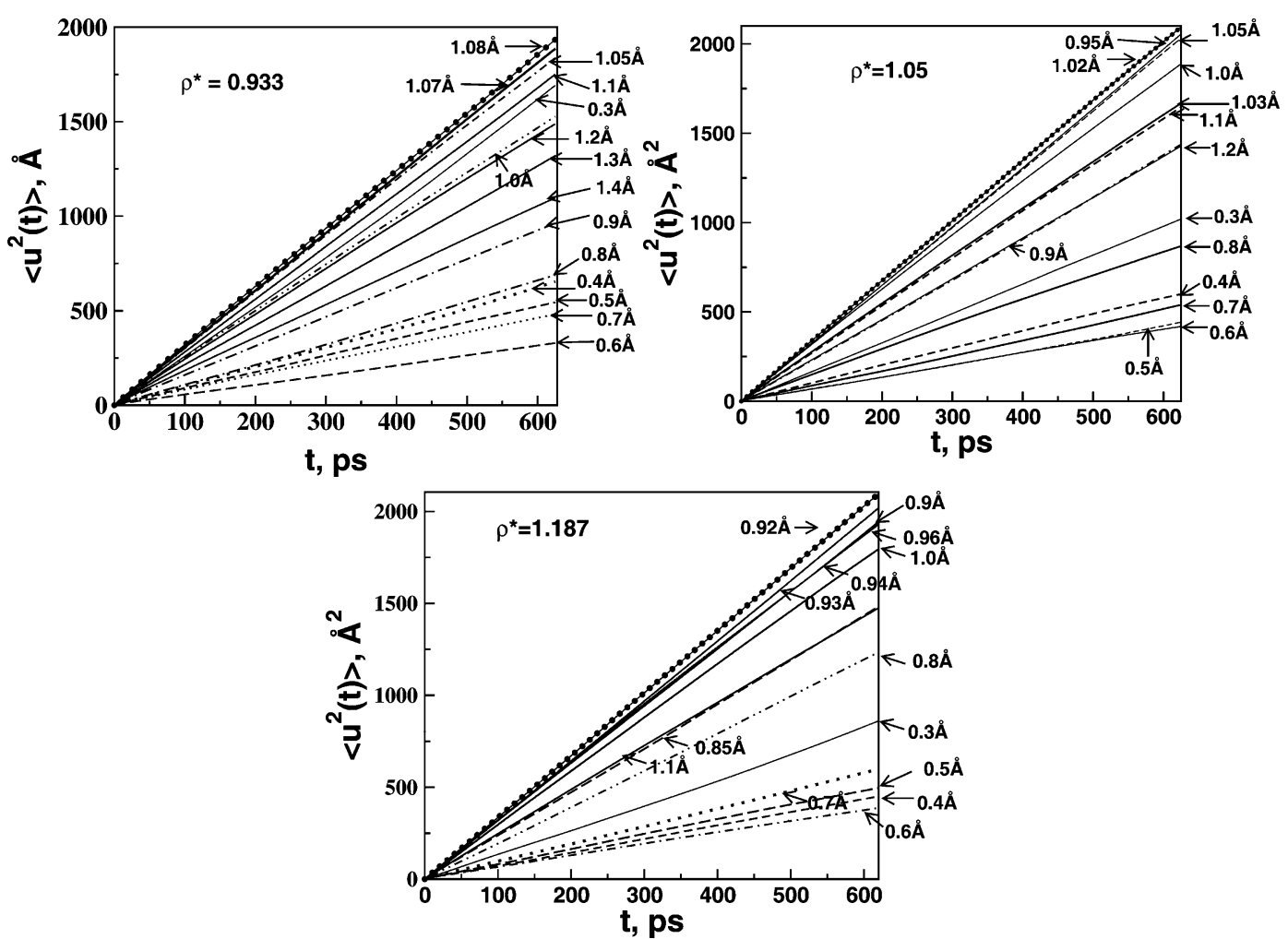

Figure 3. Mean square displacement of impurities of various sizes as a function of time at densities, $\rho^{*}=0.933$, 1.05 and 1.187.

The diffusivity, $D$ of the impurities can be obtained from the Einstein relationship between mean square displacement and self diffusivity. Initially the diffusivity decreases with increase in the impurity size but impurity size $0.7 \AA$ onwards, there is an increase in the diffusivity with maximum diffusivity for sizes $1.08,1.02$ and $0.92 \AA$ at the three reduced densities $0.933,1.05$ and 1.187 respectively. Further decrease in diffusivity is seen as the size of impurity increases. We can categorize the observed dependence 
into two regimes: (a) small impurities when the self diffusivity decreases with increase in size of impurity atoms. This is termed the linear regime (LR) : (b) large impurities, when the self diffusivity exhibits an anomalous maximum. This is the anomalous regime (AR).

We note that the observed maximum has been reported earlier for diffusants in both liquid medium and crystalline porous solids [12,17]. Two distinct regimes similar to the present results were also seen in liquid and porous crystalline phases. However, this is the first time such a maximum has been shown to exist in an amorphous phase. The existence of such maximum in amorphous solid with frozen disorder suggests that the Levitation Effect is a highly universal behaviour whose existence is determined by something more fundamental than just the nature of the phase. Further, it demonstrates that the diffusivity maximum exists irrespective of whether the atoms are frozen or mobile or ordered or disordered. To understand the observed behaviour, a dimensionless parameter, known as Levitation parameter, $\gamma$ can be defined as

$$
\gamma=\frac{\sigma_{i i} / 2}{\bar{r}_{n}}
$$

This definition of the Levitation parameter is appropriate for a crystalline solid where void and neck radii are well defined. In a disordered solid, void and neck radii have a wide distribution (as in the present study) instead of a well defined value. For such disordered systems, we propose a modified definition for the Levitation parameter

$$
\gamma=\frac{\zeta \cdot \sigma_{i i} / 2}{\bar{r}_{n}}
$$

where $\zeta$ is a dimensionless parameter that depends on the degree of disorder. For perfectly ordered solids, $\zeta=1$ and $\zeta$ decreases with increase in the degree of disorder. Although there are approaches to quantify the degree of disorder [18], these are still not very reliable and for a small system of particles, these methods only provide an approximate estimate of the amount of disorder. We therefore do not try to estimate $\zeta$ here but use earlier expression, eq. (5) for the rest of the discussion. The effect of this assumption is that the maximum in $D$ in the plot of self diffusivity against $\gamma$ will be seen at $\gamma$ less than unity instead of close to unity.

Figure 4 shows the size dependence of diffusivity with change in the density of the system. With increase in density, there is little or no change in the nature of the curve, upto an impurity size of about $0.7 \AA$. For larger sizes of the impurity, $0.8 \AA$ and beyond, the nature of the curve shows dramatic changes: the maximum in self diffusivity shifts gradually to lower impurity sizes with increase in density. Note that the range of impurity sizes whose $D$ are altered significantly are those with radii comparable to void radii; those impurity atoms whose radii are significantly smaller than void radii, namely, $\sigma_{i i}<0.6 \AA$ are least affected by change in density. 


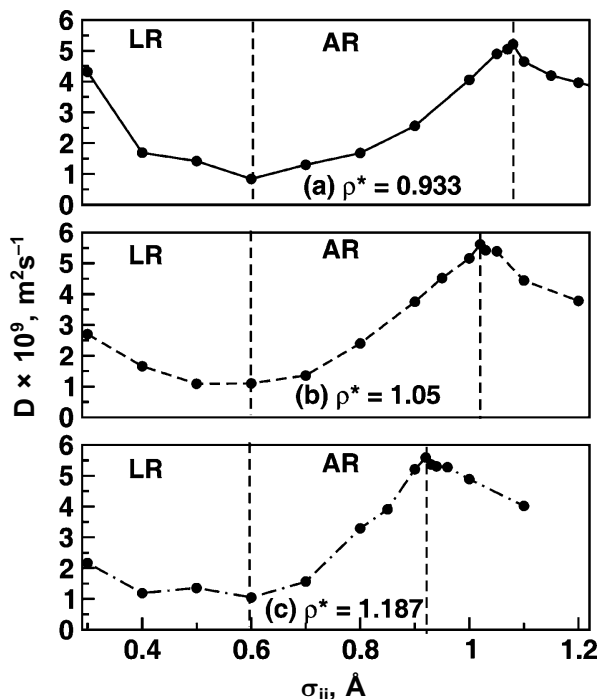

Figure 4. Variation of the self diffusivity, $D$ of the impurity atom as a function of the size of impurity atom, $\sigma_{i i}$ at different densities. Two distinct regimes can be seen : (a) when the impurity atom is small, $D$ decreases with increase in $\sigma_{i i}$ (linear regime) and (b) the anomalous increase in diffusivity starting from $\sigma_{i i}=0.7 \AA$ onwards (anomalous regime).

As the density increases, the void and neck distribution shifts to smaller values. We know that the maximum in self diffusivity occurs when the size of the diffusant is comparable to the size of the bottleneck $[12,17]$. A shift in the maximum in neck sizes seen in Figure 2 implies that the size of the diffusant at which the maximum is seen also shifts to smaller values. The magnitude of the shifts in $r_{n}$ do not correspond to the magnitude of the shifts in $\sigma_{\max }$ (the value of diffusant diameter, $\sigma_{i i}$ at which $D$ is maximum) for several reasons : (a) first, the degree of disorder (which is accounted for by the factor $\zeta$ ) also changes with density and (b) the skewed shape of $f\left(r_{n}\right)$.

A plot of $D$ against $\gamma$ is shown in Figure 5. The diffusivity maximum occurs at $\gamma=0.683,0.740$ and 0.810 for the three reduced densities, $\rho^{*} 0.933,1.05$ and 1.187 respectively. Note that $\gamma$ value tends towards unity with increase in density. We believe this is due to decrease in degree of disorder with increase in density. An increase in height of first peak in $g_{h h}(r)$ supports this view.

We have calculated several other properties to understand the behaviour more comprehensively. Figure 6 shows the velocity autocorrelation function (VACF) of the different impurity sizes for the three densities. The impurities in the linear regime experiences large backscattering while the impurity size near the maximum in self diffusivity has little backscattering. At higher densities, the impurity close to the anomalous maximum experiences even less backscattering. This is counter intuitive since at higher densities, the impurity atom should experience more collisions with the host matrix and consequently, more backscattering. However, the reasons for the lower backscattering can be understood in terms of the force exerted on the impurity by the 


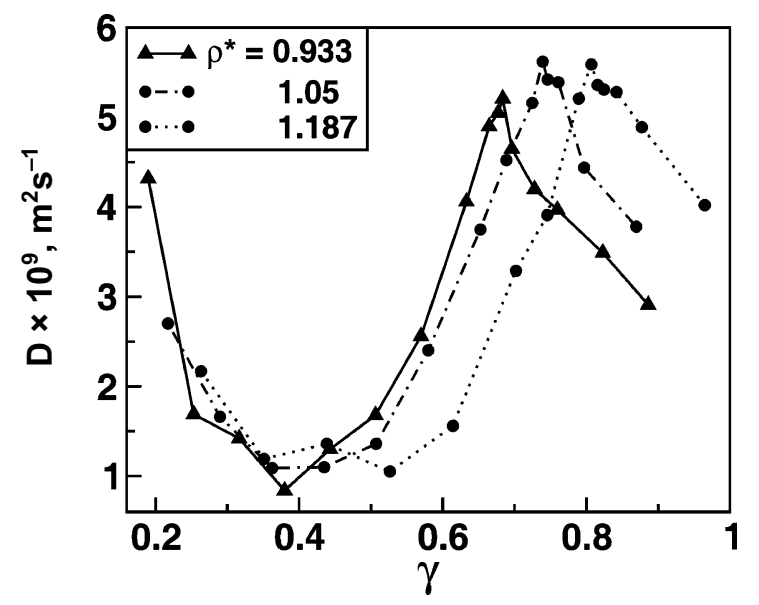

Figure 5. Variation of the self diffusivity, $D$ of the impurity atom as a function of the dimensionless Levitation parameter $\gamma$ at three different densities. We see that with increase in density the maximum shifts to larger $\gamma$ although the maximum shifts to smaller sizes of the diffusant (see Figure 4). This is probably due to increase in the degree of disorder with increase in the density.
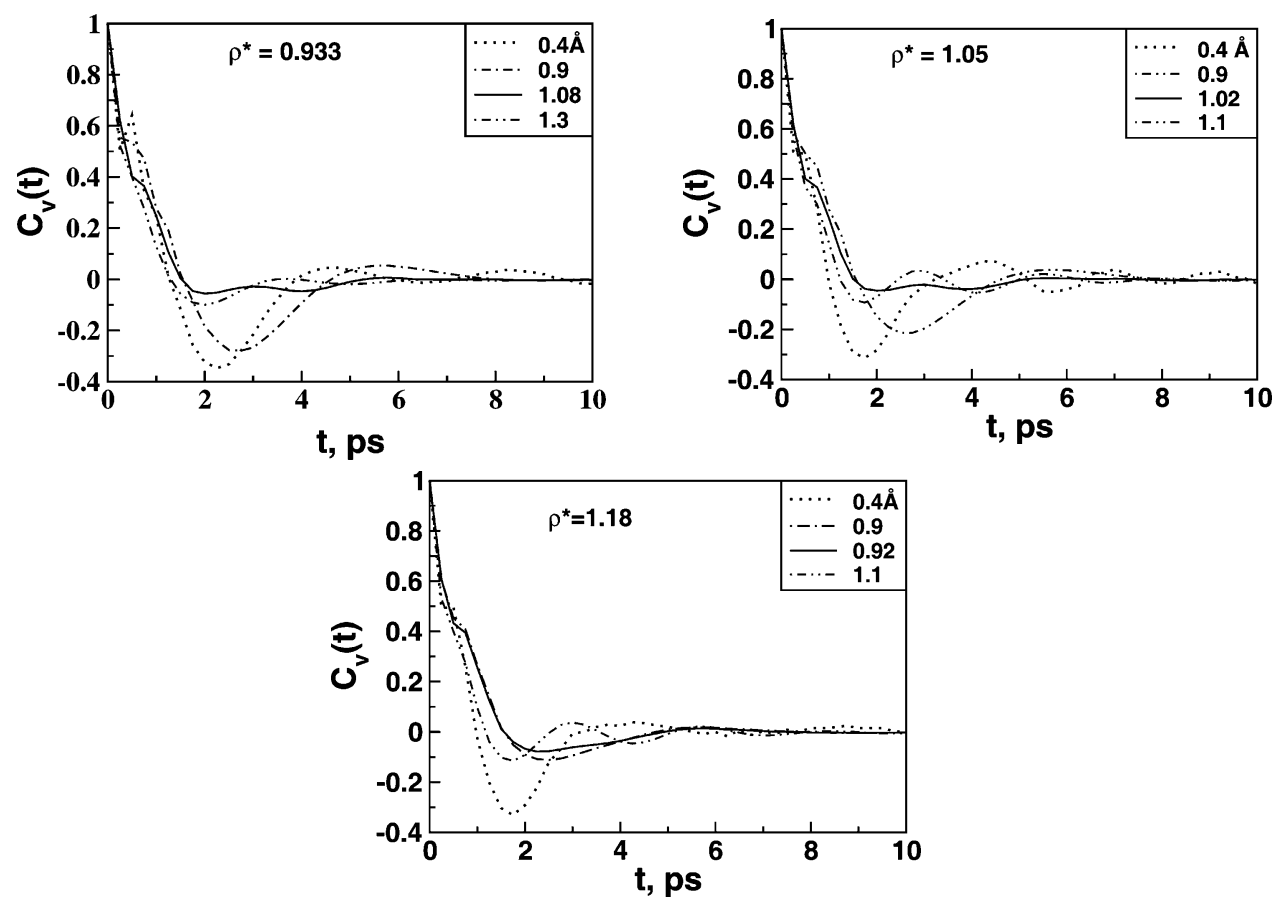

Figure 6. Velocity autocorrelation (VACF) function for various impurities belonging to the linear (LR) and 0 (AR) regimes.

host and the resulting potential energy landscape as we shall show below.

The average mean square force acting on the impurity due to the host atoms at different densities is reported in Figure 7. For all the densities, impurity with diffusivity maximum experiences minimum average mean square force. Both this and 

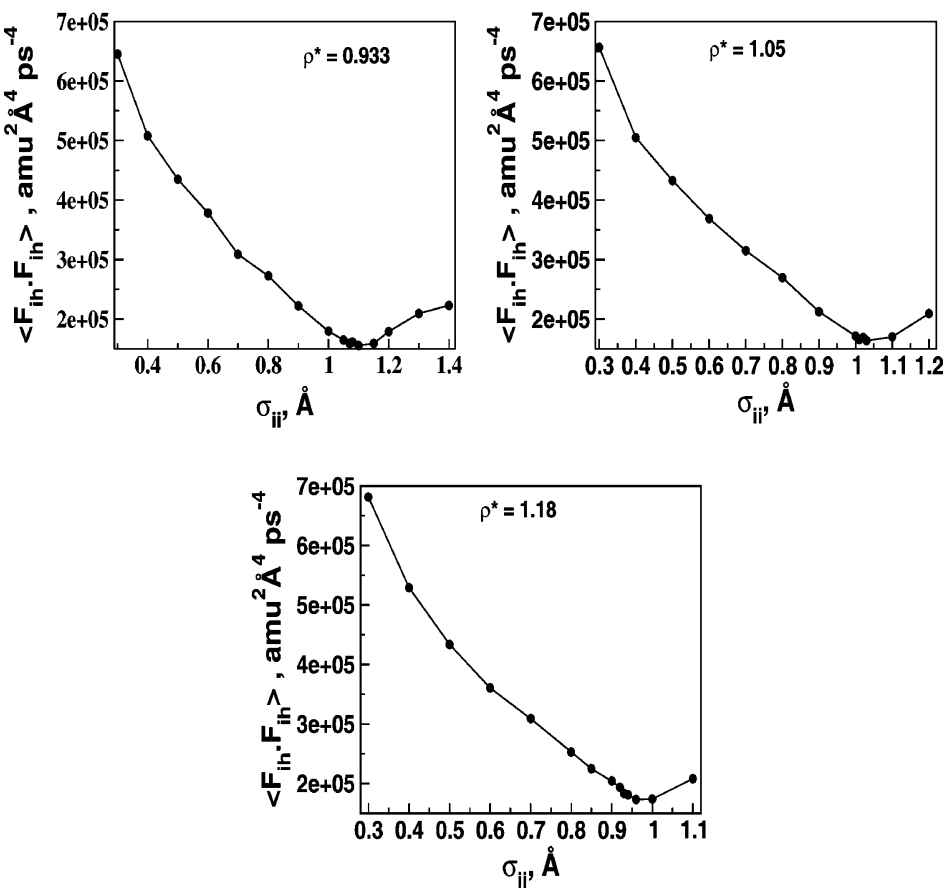

Figure 7. Average mean square force acting on impurity atoms due to neighboring host atoms as a function of the size of the impurity atom for the three densities.

the VACF are explained by the fact that when the size of the impurity is similar to that of the neck interconnecting two voids through which the impurity species passes through during its sojourn, the diffusant experiences a mutual cancellation of force. Such a cancellation occurs because, the force exerted by the medium on the diffusant from a given direction is opposite and equal to the force exerted by the medium that is diagonally opposite to it. Thus, forces cancel mutually leading to lowered mean square force. The impurity atom then exhibits maximum diffusivity since although it is inside the medium it is essentially not bound. The potential energy landscape for this impurity atom is less undulating. This follows from $\boldsymbol{F}=-\boldsymbol{\nabla} \phi$; lower force means changes in $\phi$ are small. The VACF of this impurity atom with maximum self diffusivity which experiences shallower potential energy landscape has facile motion with minimal or no backscattering.

We have previously observed similar behaviour in binary liquid mixtures [2,12]. The present host is a frozen amorphous solid. It is instructive to compare the behaviour of the VACF and other properties of the impurity species at the diffusivity maximum. In the liquid, the host atoms are also mobile and the backscattering observed is almost zero while in the present study we do find some backscattering (and less facile motion) of the impurity species at the diffusivity maximum. This suggests that even though a liquid may be more disordered (both static as well as dynamic disorder) as compared to an amorphous solid, this does not affect the motion of particle close to 
the diffusivity maximum. Further, the lower backscattering in the liquid suggests that there is cooperative motion between the impurity atom or solute and the host or solvent in the case of liquid mixtures; there can be no other explanation for the lower backscattering in the liquid phase as compared to the amorphous phase.

In Figure 8 we show Arrhenius plot of self diffusivity for an impurity from linear regime and another close to the diffusivity maximum. Large magnitude of force suggests that the potential energy landscape of the linear regime impurity has larger undulations and consequently higher activation energy. On the other hand, the impurity at the diffusivity maximum is associated with a shallower potential energy landscape and therefore a lower activation energy. We see from the figure that this is indeed the case. Previously, studies of diffusants in porous solids, liquids and ions in polar
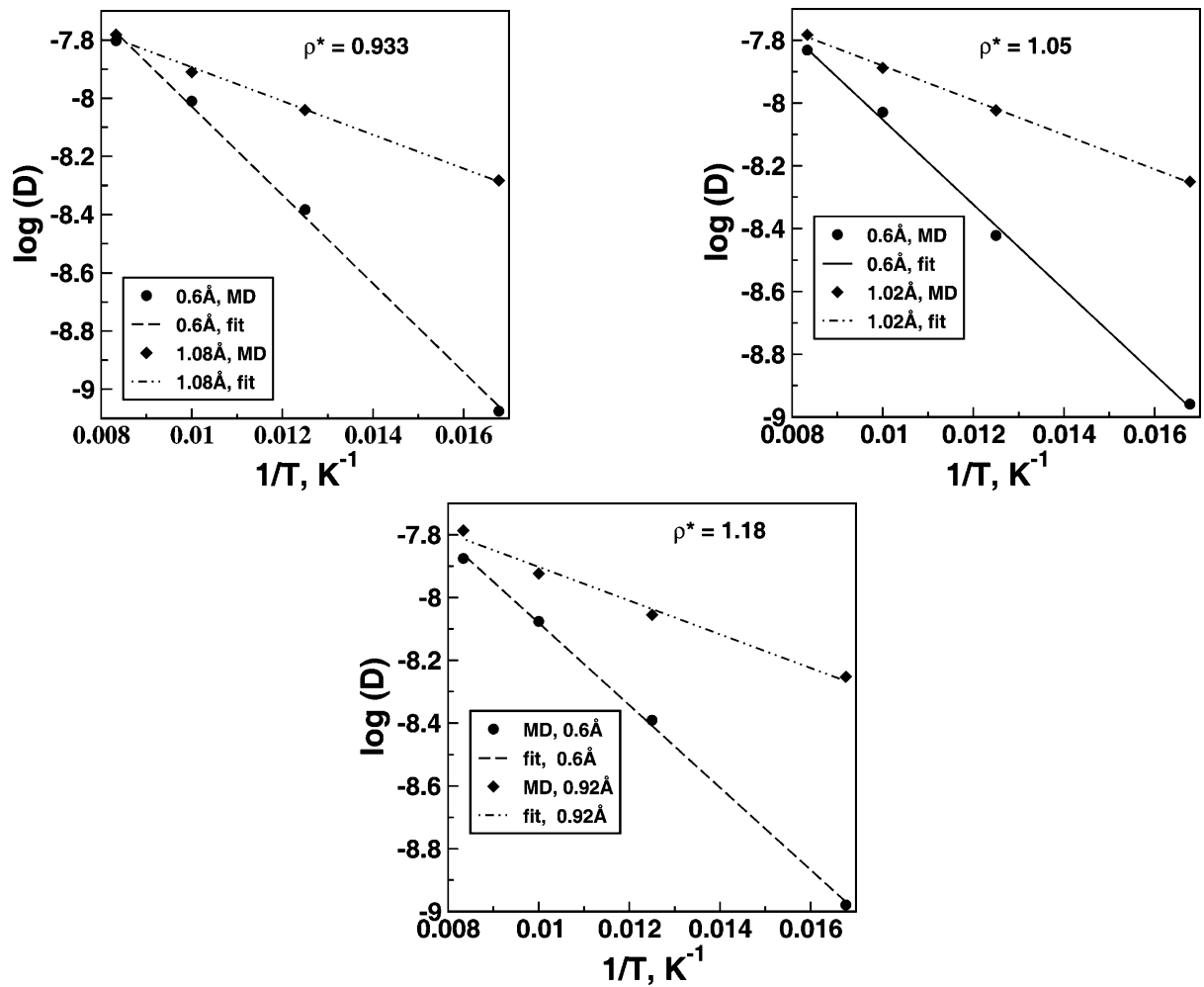

Figure 8. Arrhenius plot for the self diffusivity of impurity sizes in linear and anomalous regimes at the three densities.

solvents all show lower activation energy for the size corresponding to the diffusivity maximum [2].

In order to get a greater insight into the nature of motion, we have computed the self part of the intermediate scattering function, $F_{s}(k, t)$ for the impurity species for different sizes at all the three densities. The MD data for different wavenumbers is fitted to Kohlrausch-Williams-Watt's stretched exponential as shown in Figure 9. Table 3 lists 
Table 2. Activation energies of $L R$ and $A R$ impurities at different densities.

\begin{tabular}{cccc}
\hline Regime & $\sigma_{i i}, \AA$ & $\rho^{*}$ & $E_{\text {act }}, \mathrm{kJ} / \mathrm{mol}$ \\
\hline LR & 0.6 & 0.933 & 2.9112 \\
AR & 1.08 & 0.933 & 1.1123 \\
LR & 0.6 & 1.05 & 2.5883 \\
AR & 1.02 & 1.05 & 1.0522 \\
LR & 0.6 & 1.187 & 2.5093 \\
AR & 0.92 & 1.187 & 1.0274 \\
\hline
\end{tabular}
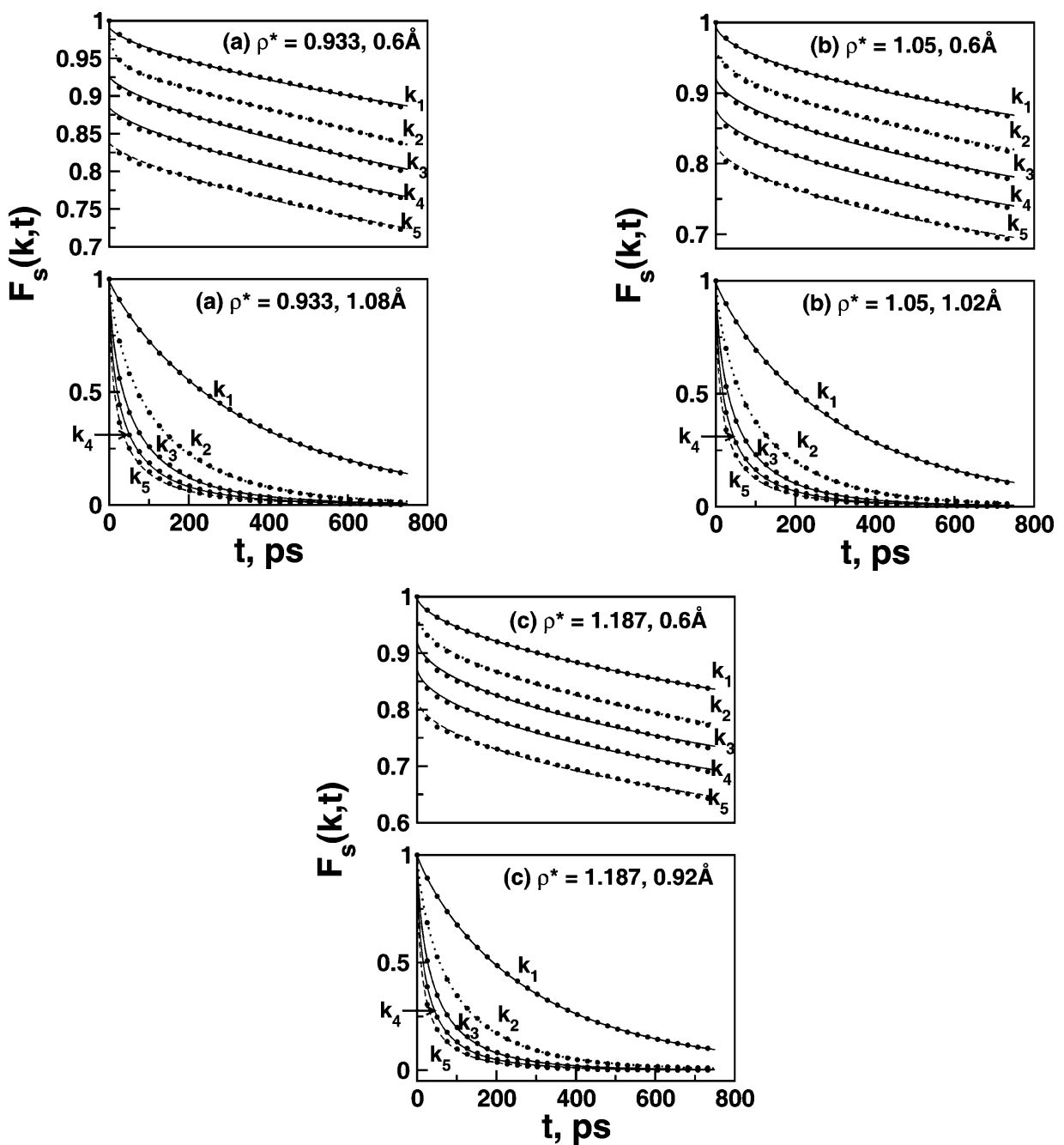

Figure 9. Self intermediate scattering plot of $L R(I)$ and $A R$ regime impurities at different densities. The filled circle is MD data and lines are the Kohlrausch-Williams-Watt's stretched exponential fit for various wavevectors, $k_{n}$. $k_{n}=2 n \pi / L$ where $n=1,2, \ldots .5$ and $L\left(\rho^{*}\right)=73.27 \AA$ (0.933), $70.44 \AA$ (1.05) and $67.61 \AA$ (1.187). The values of wavevectors and $\alpha, \beta, \tau$ are reported in Table 3 . 
Table 3. Coefficients for the Kohlrausch-Williams-Watt's stretched exponential obtained by fitting to self part of the intermediate scattering function of the impurities of different sizes for two different wavenumbers.

\begin{tabular}{cccccc}
\hline$\rho^{*}$ & $\sigma_{i i} \AA$ & $k, \AA^{-1}$ & $\alpha$ & $\beta$ & $\tau, \mathrm{ps}$ \\
\hline 0.933 & 0.6 & 0.0858 & 0.9918 & 0.6710 & 19636.3 \\
& & 0.4290 & 0.8386 & 0.7076 & 11318.0 \\
0.933 & \multirow{2}{*}{1.08} & 0.0858 & 0.9908 & 0.9157 & 358.7 \\
& & 0.4290 & 1.0677 & 0.4846 & 23.2 \\
1.05 & 0.6 & 0.0892 & 0.9960 & 0.5671 & 24933.4 \\
& & 0.4460 & 0.8293 & 0.5701 & 15823.2 \\
1.05 & \multirow{2}{*}{1.02} & 0.0892 & 0.9905 & 0.9177 & 314.3 \\
& & 0.4460 & 1.0979 & 0.4711 & 19.0 \\
1.18 & 0.6 & 0.0929 & 0.9991 & 0.5913 & 13894.0 \\
& & 0.4645 & 0.8203 & 0.5430 & 10442.7 \\
1.18 & 0.92 & 0.0929 & 0.9984 & 0.8954 & 289.5 \\
& & 0.4645 & 1.1155 & 0.4812 & 15.3 \\
\hline
\end{tabular}

the values of the parameters $\alpha, \beta$ and $\tau$ of the stretched exponential. There are several interesting points that provide additional insights into the nature of motion that may be noted from Table 3.. The relaxation time, $\tau$ is lower for AR as compared to LR impurity for a given wavenumber. This clearly shows that the density-density correlation between the impurity and the same impurity at a later time decays faster in the case of AR. This is consistent with the higher self diffusivity exhibited by AR. The value of $\beta$ depends on the nature of the medium : $\beta$ is close to unity in liquid medium while it will be significantly less than unity for amorphous solids. $\beta$ for the impurity from the AR is larger than for the LR impurity for smaller $k$ values : $0.89<\beta<0.91$ for AR while it is $0.56<\beta<0.67$ for LR. This shows that for long range motion corresponding to a distance of $2 \pi / k$ (where $k=0.0892 \AA^{-1}$ ) $=70 \AA$ the motion of the anomalous regime particle is rather facile. Further, the fact that the value of $\beta$ is close to unity for the anomalous regime impurity, suggests that the motion of this impurity deviates from the typical stretched exponential seen in the amorphous solids and tends towards usual exponential decay observed in liquids. This behaviour needs to be investigated in more detail. The present work thus demonstrates for the first time that there are similarities between diffusion in liquid and the amorphous phases under certain circumstances.

\subsection{Implication of size dependence : Dependence of self diffusivity on density :}

The observed dependence of self diffusivity on the size of the impurity atom in the amorphous matrix and the changes in this dependence with density have important consequences on the dependence of self diffusivity on density. We now take the data 
presented previously and replot them. Our analysis shows that the dependence of the self diffusivity on density is dramatically different for different impurity sizes. We discuss this here along with some experimental data.

Small-sized $(0.8 \AA)$ impurity atom : First, consider a relatively small-sized impurity, 0.8 $\AA$. In Figure 4 we see that this size is smaller than the size for which diffusivity is maximum. In Figure 10, we plot the variation of the self diffusivity of this size with

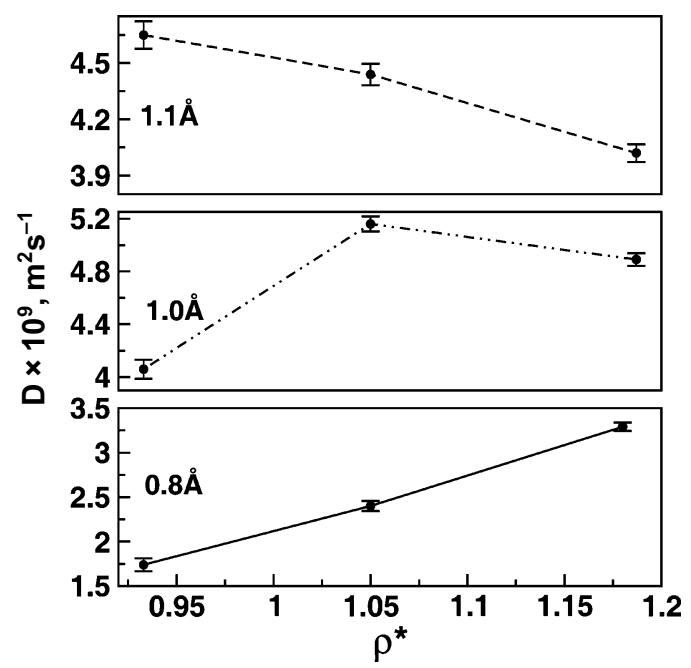

Figure 10. Diffusivity of $0.8,1.0$ and $1.1 \AA$ impurities at various densities.

increase in density. Error bars for the computed $D$ values are also indicated; note that the changes are beyond the error in the value of $D$ and therefore the observed trends are reliable. We see that with increase in density, the self diffusivity also increases. This is unexpected since kinetic theory only predicts a decrease in self diffusivity with increase in density. An increase in density is expected to lead to more frequent collisions (of the diffusant with the atoms comprising the medium) and therefore its mean free path traversed between collisions decreases. As a result, the self diffusivity is expected to decrease with increase in density. But we find that the simulations suggest a behaviour that is contrary to this. This counter intuitive result, however, can be easily understood in terms of the Levitation Effect. From the plot of $D$ with $\gamma$, we see that self diffusivity decreases with increase in $\gamma$ in the range 0 to $\sim 0.4$ (or 0.5). Between -0.4 to -0.8 , we see an increase in $D$ with $\gamma$. The dependence of $D$ with $\gamma$ is central to understanding the behaviour of $D$ with density.

For $0.8 \AA$ impurity atom, the corresponding $\gamma$ (at the range of densities we are studying) is around 0.5. An increase in density, generally leads to reduction in the sizes of the voids and necks. As a result, in the expression given by eq. (5), the value of $\bar{r}_{n}$ decreases with increase in density. This leads to an increase in $\gamma$ and consequently, the $D$ increases with density. 
Intermediate-sized $(1.0 \AA)$ impurity atom : In Figure 10 the dependence of $D$ is plotted against density for impurity size of $1.0 \AA$. We note that the $D$ value now exhibits an anomalous maximum with density. Again, we emphasize that the changes in the values of $D$ are beyond the error bars and therefore no ambiguity exists about the dependence of $D$ on density for the various sizes of the impurity atom. This behaviour can also be understood with the help of dependence of $D$ on $\gamma$. We note that $\gamma$ corresponding to $1.0 \AA$ impurity size is between 0.64 and 0.7 depending on the density. As already noted, the effect of an increase in density is to reduce the neck radius leading to increase in the value of $\gamma$ irrespective of the size of the impurity atom. We have also seen from the plot of $D-\gamma$ that for this range of $\gamma$, the value of $D$ increases and then decreases as it goes past the diffusivity maximum. Thus, the consequence of an increase in density is to increase $\gamma$ which then goes through a maximum. Thus, the impurity atom of this size has quite a different dependence on density as compared to the smaller-sized impurity atom of $0.8 \AA$ size.

Large-sized $(1.1 \AA)$ impurity atom : We now consider the effect of density on the largesized impurity atom of $1.1 \AA$ size. We see that the self diffusivity decreases with increase in the density. This is easily understood since an increase in density leads to a decrease in $\bar{r}_{n}$ and therefore an increase in $\gamma$. We have previously noted from the plot of $D$ against $\gamma$ that increase in $\gamma$ beyond the value corresponding to diffusivity maximum only leads to a decrease in $D$.

Thus, we see that for small, intermediate and large-sized impurity atoms, Levitation Effect dictates observed behaviour; the changes in neck radius vis-a-vis the impurity atom size are responsible for the observed remarkably different dependencies.

Is there experimental or simulation data that follows the results obtained from the molecular dynamics simulations here? We have previously shown [2] that the diffusivity maximum is a generic and universal behaviour seen not only in systems with LennardJones interactions but also those with electrostatic interaction. We have also shown that Levitation Effect exists in widely different phases and systems. It therefore follows that the variation of self diffusivity with density is also a generic and ubiquitous behaviour. We did refer to simulations of $M X$ and $\mathrm{MX}_{2}$ melts in the introduction. Woodcock and coworkers [10] have shown that in these systems, a maximum in diffusivity as a function of density of the constituent ions is seen at intermediate densities. Melts of $\mathrm{NaAlSi}_{2} \mathrm{O}_{6}$ also exhibited density dependent maximum in self diffusivity of ions such as $\mathrm{Si}^{4+}, \mathrm{Al}^{3+}$ as well as $\mathrm{O}^{2-}$ [11]. We suggest that these are examples of the density dependent maximum in self diffusivity demonstrated in this study. In order to confirm that such a maximum in self diffusivity as a function of density exhibited by these melts have indeed their origin in the Levitation Effect as suggested in this work, certain other properties may be computed. For example, our conceptual input here suggests that the diffusant with maximum diffusivity will also have lowest activation energy. This could easily be measured or computed to see if our suggestion is true. 


\section{Conclusions}

It is evident that different sized impurity atoms exhibit completely and dramatically different dependence on density. A logical explanation for this behaviour has been provided in terms of the Levitation Effect. We said that large-sized impurity atoms will exhibit the expected decrease in $D$ with $\rho$. It could be asked what is large? In other words, what is the size that may be considered large and for which size $D$ will decrease with $\rho$ ? Similarly, what sizes may be considered intermediate and what sizes may be said to be small? How does one determine the boundaries between small and intermediate and between intermediate and large? This is an important question.

Firstly, we note that the precise range for small, intermediate and large size is determined by the void structure of the host matrix. An impurity atom may be said to be small $\left(\sigma_{i i}<2 \bar{r}_{n}\right)$, if the impurity atom is smaller than the neck diameter of the host void structure. However, note that if the impurity atom radius is much smaller $\left(\sigma_{i i}<<2 \bar{r}_{n}\right)$ than the neck radii, then it might lie in linear regime (see Figure 4) where increase in density and therefore $\gamma$ will lead to decrease in $D$. This behaviour is same as what is seen for large-sized impurity atoms. An intermediate-sized impurity atom is one that is close to the size of the neck radius in the host matrix. Finally an impurity atom is large in size if its size is larger than the size of the neck radii.

We also explained the observed behaviour of $D$ with $\gamma \cdot \gamma$, the Levitation parameter is most strongly influenced by the void and neck distributions. In defining the Levitation parameter $\gamma$, only the average neck radius is considered; no account of the changes in the distribution (broad, narrow or skewed) are included in defining $\gamma$. As a result, at times, changes in $\gamma$ alone can provide only a first-order influence on $D$. A weaker, albeit important influence discussed here is sometimes necessary to be taken into account to explain the experimentally observed trend. In the solids, the range over which the density can be varied is limited and therefore changes in $D\left(\rho^{*}\right)$ behaviour with size of the diffusant can be difficult to observe. It should be borne in mind that the degree of disorder included through the factor $\zeta$ will also influence the value of $D$.

We note that Levitation Effect is indeed a generic effect that is ubiquitous. It has been seen in liquids where the neighbouring hosts are as mobile as the impurity or solute. This suggests that the effect does not disappear in spite of both static and dynamic disorder seen respectively in amorphous solids and liquids. Here the free energy landscape picture discussed by us [2] or by Odagaki and coworkers [19] is more appropriate since the free energy landscape in a liquid and an amorphous solid are not qualitatively different.

\section{Acknowledgment}

Authors wish to thank Department of Science and Technology, New Delhi and CSIR, New Delhi, for financial support in carrying out this work. Authors also acknowledge CSIR, New Delhi for a research fellowship to MS. 


\section{References}

[1] Y Zhou and G H Miller Physical Review E53 1587 (1996)

[2] S Yashonath and P K Ghorai J. Phys. Chem. B112 665 (2008)

[3] D Kivelson, S K Jensen and M-K Ahn J. Chem. Phys. 58428 (1973)

[4] B Alder and W E Alley J. Chem. Phys. 611415 (1974)

[5] G Tarjus and D Kivelson J. Chem. Phys. 1033071 (1995)

[6] Y Jung, J P Garrahan and D Chandler Physical Review E69 061205 (2004)

[7] A Pradel, T Pagnier an M Ribes Solid State lomics 17147 (1985)

[8] M Hosono, J Kawamura, H Itoigawa, N Kuwata, T Kamiyama and Y Nakamura J. of Non-Crystalline Solids 24481 (1999)

[9] T Minami J. of Non-Crystalline Solids 73273 (1985)

[10] L V Woodcock, C A Angell and P Cheeseman J. Chem. Phys. 651565 (1976)

[11] C A Angell, P A Cheeseman and S Tamaddon Science 218885 (1982)

[12] P K Ghorai and S Yashonath J. Phys. Chem. B109 5824 (2005)

[13] M Sharma and S Yashonath J. Phys. Chem. B110 17217 (2006)

[14] M Parrinello, A Rahman and P Vashishtha Phys. Rev. Lett. 501073 (1983)

[15] P Vashishtha and A Rahman Phys. Rev. Lett. 401337 (1978)

[16] The dl-poly-2.13. W Smith and T Forester CCLRC, Daresbury Laboratory, Daresbury (2001)

[17] S Yashonath and P Santikary J. Phys. Chem. 986368 (1994)

[18] S Yashonath and V C Bhasu J. Chem. Phys. Lett. 189311 (1992)

[19] T Yoshidome, A Yoshimori and T Odagaki Phys. Rev. E76 021506 (2007) 